\title{
Response to Owen, Singh and Kirschner - Prenatal testing is a necessary part of antenatal care
}

\section{LARS H BREIMER}

Keywords: Non-invasive prenatal testing, disability, antenatal care

Everyone wants a healthy baby. No sane person sets out to have a sick or disabled child. It is the duty and joy of healthcare to help to increase the chances of a happy event. Until delivery, healthcare must do its utmost to decrease the risk of a sick child or a child with a disability being born.

If that was not clear to the authors of the commentary (1) from my article (2), then it was a shortcoming and a pity.

Non-invasive prenatal testing (NIPT) should be viewed as an additional tool to increase the chances of a healthy outcome. This test is part of the armamentarium of modern antenatal care. Actions to help increase the chances of a child being born healthy and in full vigour cannot be seen as a harm. To claim that to prevent a disability before birth would cause harm would be a perversion of language.

NIPT is evolving. Like all new technologies, we have to learn to use it optimally, but it is with us whatever we choose. Nevertheless, in principle, NIPT is no different from other technologies used to maximise the chances of having a healthy baby. All the pregnant should have the right to the best technology.

In most countries, NIPT is not part of the standard antenatal service. The pregnant must turn to the private sector, where the price is usually much higher than the real cost. Nevertheless, it is clear that even people with limited means choose NIPT. In

Author: Lars H Breimer (lars.breimer@regionorebrolan.se), School of Health and Medical Sciences, Örebro University, and Consultant Physician, Department of Laboratory Medicine, Clinical Chemistry, Örebro University Hospital, S-701 85 Örebro, SWEDEN.

To cite: Breimer LH. Response to Owen, Singh and Kirschner - Prenatal testing is a necessary part of antenatal care. Indian J Med Ethics. 2020 OctDec; 5(4) NS: 294. DOI: 10.20529/IJME.2020.113.

(c) Indian Journal of Medical Ethics 2020
The Netherlands, where NIPT is provided at the same price as first trimester combined screening (FTC), NIPT has wiped out FTC. Belgium is the only country that I know of which provides NIPT at a token cost ( $\$ 9$, the same as any blood test).

The studies I quoted (2) show that when the burden of caring for a child with disability falls on family members, people vote with their feet and wallets. In a stressed system, health insurers and public providers might take the step of giving the option of a free NIPT with the proviso that if this is declined or persons do not act on the results, the cost of care of any offspring with disability will not be covered, but rather fall on the parents.

The authors have not declared any conflicts of interest. The authors' third reference is to an article of mine, where I with my co-authors, Nilsson and Breimer, point out that declarations of conflicts of interest are still inadequate. The authors have done an enormous amount of work to improve conditions for persons with disabilities. It is, therefore, surprising that they seem not to support reducing the number of persons born with disabilities. One senses a possible undeclared agenda here. To avoid a disability ought to be preferable to coping with it. Also, the fewer the persons with disabilities, the more funding is available for each in a financially-constrained system.

Most if not all religions and life philosophies warn those in authority - religious and official — not to put heavy burdens on the backs of others, burdens that the officials themselves would not lift a finger to help bear, and which they certainly would not accept for themselves.

Everyone hopes for a healthy baby, and the purpose of antenatal care, which now includes NIPT, is to maximise the chances of this. If that was not clear enough from my article, that is a pity. Or do the three authors have any other stance on this point that is the cause of their opposition to my paper (even if they never explain why)?

\section{References}

1. Owen A, Singh S, Kirschner K. Disability activism and non-invasive prenatal testing: A response to Breimer. Indian J Med Ethics. 2020 OctDec;5(4) NS:290-3.DOI: 10.20529/JME.2020.112.

2. Breimer LH. Non-invasive prenatal testing: Special interest groups vs women's autonomy. Published online first on June 20,2020. Doi: https:// doi.org/10.20529/IJME.2020.069 